\title{
Involvement of aspartoacylase in tremor expression in rats
}

\author{
Ai NISHITANI ${ }^{1)}$, Miyuu TANAKA ${ }^{1)}$, Saki SHIMIZU ${ }^{2)}$, Naofumi KUNISAWA ${ }^{2)}$, Mayuko YOKOE ${ }^{1)}$, \\ Yusaku YOSHIDA ${ }^{3)}$, Toshiro SUZUKI ${ }^{3)}$, Tetsushi SAKUMA ${ }^{4)}$, Takashi YAMAMOTO ${ }^{4)}$, \\ Mitsuru KUWAMURA ${ }^{5)}$, Shigeo TAKENAKA ${ }^{6}$, Yukihiro $\mathrm{OHNO}^{2)}$, and Takashi KURAMOTO ${ }^{1)}$ \\ 1) Institute of Laboratory Animals, Graduate School of Medicine, Kyoto University, Yoshida-konoe-cho, Sakyo-ku, \\ Kyoto 606-8501, Japan \\ ${ }^{2)}$ Laboratory of Pharmacology, Osaka University of Pharmaceutical Sciences, Takatsuki 569-1094, Japan \\ ${ }^{3)}$ Research Department for Experimental Animals, Biotechnical Center Japan SLC, Inc., Aoihigashi, Naka-ku, \\ Hamamatsu, Shizuoka 433-8114, Japan \\ 4) Department of Mathematical and Life Sciences, Graduate School of Science, Hiroshima University, 1-3-1 \\ Kagamiyama, Higashi-Hiroshima, Hiroshima 739-8526, Japan \\ ${ }^{5)}$ Laboratory of Veterinary Pathology, Osaka Prefecture University, Izumisano, Osaka 598-8531, Japan \\ ${ }^{6)}$ Laboratory of Molecular Biology, Osaka Prefecture University, Izumisano, Osaka 598-8531, Japan
}

\begin{abstract}
Essential tremor (ET) is a common movement disorder with a poorly understood etiology. The TRM/Kyo mutant rat, showing spontaneous tremor, is an animal model of ET. Recently, we demonstrated that tremors in these rats emerge when two mutant loci, a missense mutation in the hyperpolarization-activated cyclic nucleotide-gated potassium channel 1 ( Hcn1) and the tremor (tm) deletion, are present simultaneously. However, we did not identify which gene within the $t m$ deletion causes tremor expression in TRM/Kyo rats. A strong candidate among the 13 genes within the $\mathrm{tm}$ deletion is aspartoacylase (Aspa), because some Aspa-knockout mouse strains show tremor. Here, we generated Aspa-knockout rats using transcription activator-like effector nuclease technology and produced AspalHcn 1 double-mutant rats by crossing Aspa-knockout rats with $H c n 1$-mutant rats. The Aspa-knockout rats carried nonsense mutations in exon 4; and ASPA proteins were not detectable in their brain extracts. They showed elevated levels of $\mathrm{N}$-acetyl-L-aspartate (NAA) in urine and spongy vacuolation and abnormal myelination in the central nervous system, but no tremor. By contrast, AspalHcn1 double-mutant rats spontaneously showed tremors resembling those in TRM/Kyo rats, and the tremor was suppressed by drugs therapeutic for ET but not for parkinsonian tremor. These findings indicated that the lack of the Aspa gene caused tremor expression in TRM/Kyo rats. Our animal model suggested that the interaction of NAA accumulation due to ASPA deficiency with an unstable neuronal membrane potential caused by HCN1 deficiency was involved in tremor development.
\end{abstract}

Key words: aspartoacylase, essential tremor, Hcn1, rat, TALEN

\section{Introduction}

Essential tremor (ET) is one of the most common movement disorders. The major manifestation of ET is a postural and/or kinetic tremor, which predominantly affects the hands, head, and vocal organs [4]. The etiology of ET, however, remains poorly understood. Certain environmental factors may be at least partly involved in

(Received 17 January 2016 / Accepted 8 March 2016 / Published online in J-STAGE 30 March 2016)

Address corresponding: T. Kuramoto, Institute of Laboratory Animals, Graduate School of Medicine, Kyoto University, Yoshidakonoe-cho, Sakyoku, Kyoto 606-8501, Japan

Supplementary video: refer to J-STAGE: https://www.jstage.jst.go.jp/browse/expanim

C2016 Japanese Association for Laboratory Animal Science 
nonfamilial ET [14]. However, a major role for genetic factors has also been suggested in the development of ET [9]. Animal models of genetic disorders and genetically manipulated animals play important roles in finding genes involved in ET development.

We recently established the TRM/Kyo rat as an animal model of ET [21]. TRM/Kyo rats show a spontaneous kinetic tremor, and their tremors are selectively inhibited by drugs therapeutic for ET but not for parkinsonian tremor. TRM/Kyo rats are double mutants for a missense mutation (c. $1061 \mathrm{C}>\mathrm{T}$, p. A354V) in the hyperpolarization-activated cyclic nucleotide-gated potassium channel 1 gene $\left(H c n 1^{A 354 V}\right)$ and tremor $(\mathrm{tm})$ gene deletion. The results of our electrophysiological experiments indicated that $H c n 1^{A 354 V}$ is a loss-of-function mutation. The tremors in TRM/Kyo rats emerge only when the two mutant loci are present simultaneously; neither rats homozygous for the Hcnl mutation nor rats homozygous for the $t m$ deletion show tremors.

The $t m$ deletion spans a nearly $240-\mathrm{kb}$ genomic region on rat chromosome 10, of which 13 genes have been mapped. The 13 genes consist of sedoheptulokinase $(S h p k)$, transient receptor potential vanilloid 1 and 3 (Trpv1/3), aspartoacylase (Aspa), spermatogenesis associated 22 (Spata22), seven olfactory receptor genes (Olr1466, Olr1467, Olr1468, Olr1469, Olr1470, Olr1471, Olr1472), and one hypothetical gene (LOC100359760) [8]. We did not identify which gene among these 13 is causative for the development of tremor in the rat model of ET. However, it is likely that the Aspa deletion may be causal because the Aspa gene is strongly expressed in the brain and several Aspaknockout mouse strains show tremor [16, 28].

We generated Aspa-knockout rats using transcription activator-like effector nuclease (TALEN) technology and produced Aspa/Hcn 1 double-mutant rats by crossing the Aspa-knockout rats with the Hcn1-mutant rats. The double-mutant rats spontaneously showed tremor resembling that of TRM/Kyo rats, and the tremor was suppressed by drugs for treating ET but not those for treating parkinsonism.

\section{Materials and Methods}

\section{Ethical use of animals}

All animal experiments were approved by the Animal Research Committees of Kyoto University and Osaka University of Pharmaceutical Sciences and were con- ducted according to their regulations on animal experimentation.

TALEN plasmid construction and messenger RNA preparation

Platinum TALEN plasmids were constructed using the two-step Golden Gate cloning method as described previously [23]. The Aspa TALEN target sequence, located in exon 4 of the Aspa (NM_024399) gene, is indicated in Fig. 1. The TALEN mRNA was synthesized from plasmids linearized by $\mathrm{XhoI}$ digestion and purified as previously described [22].

\section{Microinjection of TALEN mRNA}

TALEN mRNA was suspended in RNase-free water at $10 \mathrm{ng} / \mu 1$. Approximately $2-3 \mathrm{pl}$ of capped mRNA was microinjected into the male pronuclei of zygotes collected from F344/NSlc rats. Embryos were transferred to the oviducts of pseudopregnant Sprague Dawley female rats [15].

\section{Quantitative PCR}

Messenger RNA was isolated using ISOGEN II (Nippon Gene Co., Ltd., Tokyo, Japan) according to the manufacturer's instructions. Real-time PCR was performed using the Thermal Cycler Dice Real Time system (Takara Bio Inc., Otsu, Japan) with SYBR Premix Ex Taq II (Takara Bio Inc.). Primer sequences for quantification of Aspa cDNA were 5'-GGCTCCATTACCCTGCTCTGTTTA-3' and 5'-CGTGATGCTGAGGACCAACTTCTA- $3^{\prime}$.

By monitoring the amplification curves of a test sample and reference samples that contained $10^{1}$ to $10^{6}$ molecules of the gene of interest, the number of target molecules in the test sample was analyzed. The number of target molecules was normalized to that of peptidylprolyl isomerase A (Ppia) as an internal control [12].

\section{Western blotting}

Brains were homogenized in an ice-chilled buffer containing $50 \mathrm{mM}$ Tris, $5 \mathrm{mM}$ EDTA, $120 \mathrm{mM} \mathrm{NaCl}$, and protease inhibitors (Complete Mini EDTA-free, Roche Diagnostics, Basel, Switzerland) using a Dounce tissue grinder. Homogenates were centrifuged at 165,000 $\times g$ for $60 \mathrm{~min}$. Precipitations were lysed with a buffer containing $50 \mathrm{mM}$ Tris, $5 \mathrm{mM}$ EDTA, $120 \mathrm{mM} \mathrm{NaCl}$, and $1 \%$ sodium dodecyl sulfate. The lysate was electrophoresed on a $10 \%$ Bis-Tris gel in $1 \times$ MOPS buffer. Blots 
A Rat Aspagene

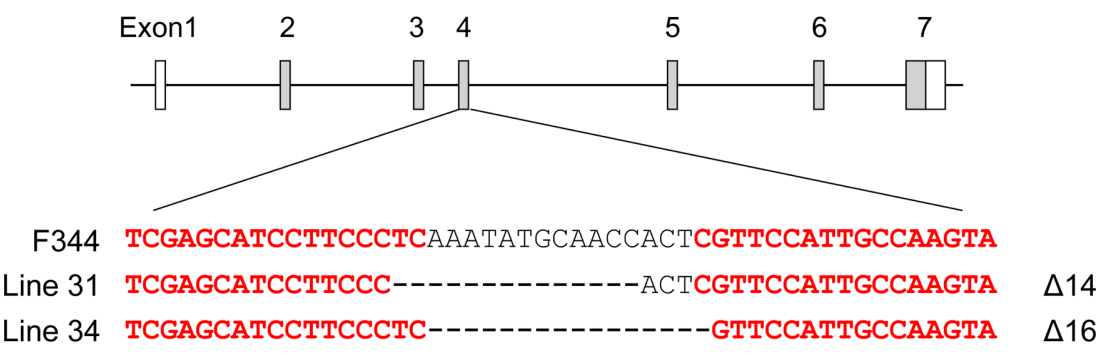

B
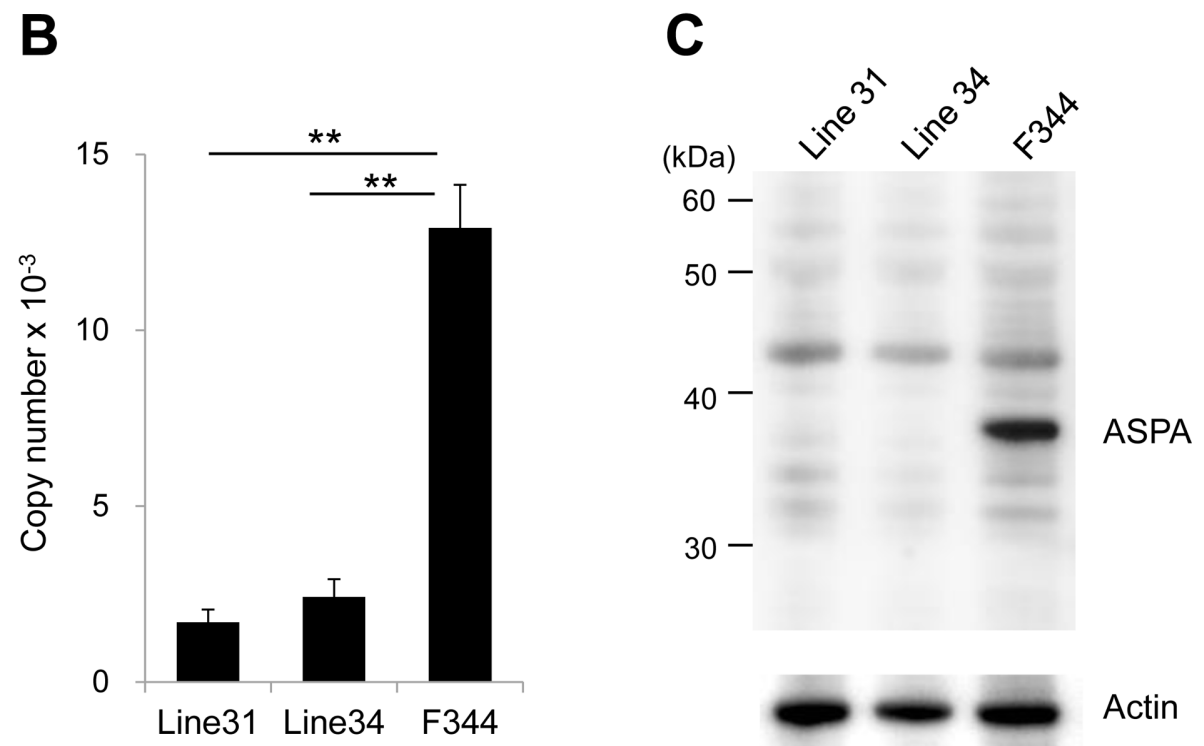

Fig. 1. Generation of Aspa-knockout rats using TALEN technology. A, Schematic representation of the rat aspartoacylase (Aspa) gene and target sites of transcription activator-like effector nuclease (TALEN). Exons are represented by boxes, and coding regions are shown in gray. TALEN target sites were designed in exon 4 of the rat Aspa gene and are shown in red. The 14-bp and 16-bp deletions are found in Line 31 and Line 34, respectively. B, Expression levels of Aspa mRNA in brains of Aspa-knockout lines and F344 rats. Expression levels are presented as copy numbers that are normalized to those of an internal control gene (Ppia). Both Aspa-knockout lines show significantly reduced expression levels compared with the control. $* * P<0.01$. C, Western blots of brain lysates from Aspa-knockout lines and F344 rats. The ASPA protein $(36 \mathrm{kDa})$ is detected in F344 but not in either Aspa-knockout line. Actin is used as a loading control.

were incubated with primary antibody in Can Get Signal Immunoreaction Enhancer Solution (Toyobo, Osaka, Japan). The Anti-ASPA antibody was produced in-house by immunizing rabbits using the ASPA peptide (SEDLPYEVRRAQEINHLFG) as an antigen. This peptide corresponded to 19 amino acids, from 83 to 101 (inclusive), of the human ASPA (NP_001121557) and was identical to the corresponding region of the rat ASPA (NP_077375). The anti-ASPA antibody was used at a dilution of 1:5,000, and the anti-actin antibody (A3853; Sigma-Aldrich, St. Louis, MO, USA) was used at 1:5,000. The secondary antibody was an anti-rabbit IgG horseradish peroxidase (GE Healthcare, Buckinghamshire, UK) and was used at 1:100,000 in Can Get Signal Immunoreaction Enhancer Solution. Signals were developed by ECL western blotting detection reagents (GE Healthcare) and detected using an image analyzer LAS3000 (Fuji Film, Tokyo, Japan).

\section{Measurement of $\mathrm{N}$-acetyl- ${ }_{-}{ }^{-}$aspartate}

Rats were housed in metabolic cages after physiological saline was orally administered at $2.5 \mathrm{ml} / 100 \mathrm{~g}$ (body weight). Urine samples from rats were collected $6 \mathrm{~h}$ later. The $\mathrm{N}$-acetyl ${ }_{\mathrm{L}}$-aspartate (NAA) level in urine was 
measured using gas chromatography mass spectrometry (GCMS) as previously described [10]. Briefly, $25 \mu 1$ of urine was mixed with $5 \mu 1$ of $1 \mathrm{mg} / \mathrm{ml}$ 2-isopropylmalic acid (Sigma-Aldrich) as an internal standard. The mixture was treated with urease to decompose and remove excess urea. After centrifuging the samples, the collected supernatant was dried under an $\mathrm{N}_{2}$ gas stream and resolved in $\mathrm{N}, \mathrm{O}$-bis (trimethylsilyl) trifluoroacetamide with $10 \%$ trimethylchlorosilane (Thermo Scientific, Tokyo, Japan). The GCMS analysis was performed using a GCMS-QP2010 Plus system (Shimadzu Corporation, Kyoto, Japan) with a DB-5 column (Agilent Technologies, Santa Clara, CA, USA).

\section{Generation of rats homozygous for both Aspa and Hcnl mutations}

The WTC rats carry the Hcn 1 missense mutation homozygously [21]. We intercrossed (WTC/Kyo $\times$ F344Aspa ${ }^{\text {em34Kyo }}$ ) $\mathrm{F}_{1}$ hybrids to obtain $\mathrm{F}_{2}$ progeny. After weaning, genotypes of Aspa and Hcnl loci were determined with the Amp-FTA method [19]. Briefly, blood samples obtained from a tail biopsy were fixed onto an FTA card (GE Healthcare), and punched out disks were used for templates. PCR was conducted with Ampdirect buffer (Shimadzu Corporation).

\section{Histology}

For histological analysis, brains and thoracic spinal cords were harvested from 12-week-old Aspa-knockout and control F344 rats. Tissue samples were fixed in 10\% neutral-buffered formalin and embedded in paraffin. Sections $4-\mu \mathrm{m}$ thick were cut and stained with hematoxylin and eosin. The thoracic spinal cords were also post-fixed with $1 \%$ osmium tetroxide at $4{ }^{\circ} \mathrm{C}$ overnight, and embedded in epoxy resin. Semi-thin $(1 \mu \mathrm{m})$ sections were cut and stained with toluidine blue.

\section{Evaluation of effects of anti-tremor agents}

Evaluating the effects of anti-tremor agents was performed as described previously [21]. Briefly, Aspa/Hcn1 double-mutant ( $8-10$ weeks of age, $n=9)$ were administered the $\beta$ receptor antagonist propranolol $(30 \mathrm{mg} / \mathrm{kg}$ ip; Sigma-Aldrich), the $\mathrm{GABA}_{\mathrm{A}}$ receptor stimulant phenobarbital (Phenobal, $20 \mathrm{mg} / \mathrm{kg}$ ip; Daiichi Sankyo Co., Ltd., Tokyo, Japan), or the muscarinic acetylcholine receptor antagonist trihexyphenidyl (3 mg/kg ip; SigmaAldrich). To evaluate tremor, animals were placed individually in an observation box $(25 \times 42 \times 20 \mathrm{~cm})$.
Tremor duration and intensity were estimated in 1-min observation periods immediately before and 15, 30, 45, and $60 \mathrm{~min}$ after drug administration. Tremor intensity was evaluated using a four-point ranked scale: 0 , none; 1 , weak (weak tremor in limited regions such as forelimbs and neck); 2, moderate (apparent tremor in extended regions such as upper body trunk including head); 3 , marked (intensive tremor in whole body).

\section{Statistical analysis}

Data are expressed as the mean $\pm \mathrm{SEM}$. The statistical significance of differences among multiple groups was determined using one-way analysis of variance and Tukey's post hoc test (for parametric data) or the Kruskal-Wallis test followed by the Steel-Dwass post hoc test (for nonparametric data). Comparisons between two groups only were determined by Student's $t$-test. A $P$ value of less than 0.05 was considered statistically significant.

\section{Results}

\section{Generation of Aspa-knockout rats}

Of 380 TALEN-injected embryos, 306 (80.5\%) were transferred to the oviducts of pseudopregnant female rats. Of these embryos, 84 (27.5\%) successfully reached the end of normal gestation, and 56 rats were weaned. Sequence analysis of the TALEN target site of these 56 founder animals revealed that three males and one female (7.1\%) carried mutations. Among them, two founder animals (\#31 and \#34) were used to develop the Aspaknockout lines. The F344-Aspa ${ }^{\text {em31Kyo }}$ that was derived from line \#31 carried a 14-bp deletion, and the F344Aspa ${ }^{\text {em } 34 K y o}$ that was derived from line \#34 carried a 16-bp deletion in exon 4 (Fig. 1A). Both deletions overlapped the TALEN target site and were deduced to generate premature stop codons as a consequence of frame shifting.

The expression levels of the Aspa mRNA in the brains of both lines decreased significantly compared with those in the control F344/NSlc rats (Fig. 1B). The western blotting results showed no expression of ASPA proteins in the brains of either line (Fig. 1C). These findings indicated that both lines had null mutations of Aspa. Thus, we used the F344-Aspa ${ }^{\text {em } 34 K y o}$ rats that carried a 16-bp deletion in further experiments. 

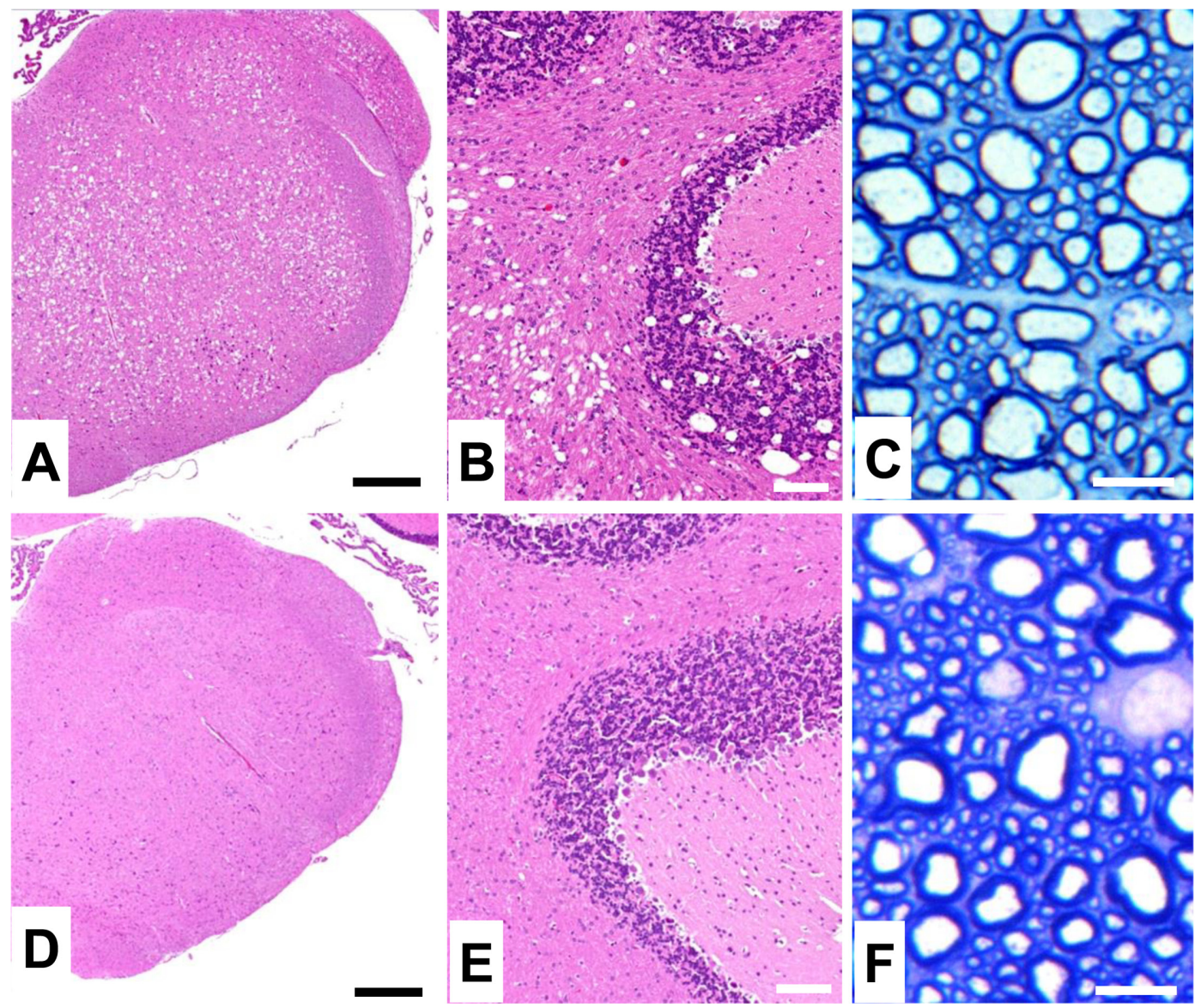

Fig. 2. Histopathology in the central nervous system (CNS) of Aspa-knockout rats. Hematoxylin and eosin- and toluidine blue-stained sections of the medulla (A, D), cerebellum (B, E) and thoracic spinal cord (C, F) in Aspa-knockout (A-C) and control F344 rats (D-F) at 12 weeks of age. In Aspa-knockout rats, severe vacuole formation (A, B) and mild hypomyelination with slightly swollen axons (C) are observed in the CNS. Scale bars: $500 \mu \mathrm{m}$ (A, D); $100 \mu \mathrm{m}$ (B, E); $10 \mu \mathrm{m}$ (C, F).

\section{Characteristics of Aspa-knockout rats}

The lack of ASPA leads to the accumulation of its substrate NAA in the brains or urine of humans and mice [17]. To measure concentrations of NAA, we collected urine from $A s p a$-knockout rats and found a significantly elevated NAA concentration of $655.2 \pm 87.9 \mu \mathrm{M}$ (mean \pm SEM). By contrast, NAA could not be detected in wildtype F344 rats.

The lack of ASPA also leads to hypomyelination and vacuolation in the central nervous system (CNS). We found that Aspa-knockout rats showed vacuole formation throughout the CNS, sometimes with a spongy appearance. Severe lesions were detected in the brainstem, midbrain, and cerebellum (Figs. 2A and B). Mild hypomyelination with slightly swollen axons was also observed in the spinal cord (Fig. 2C). These histopathological features were similar to those found in the TRM/
Kyo rats. In some previous studies, Aspa-knockout mouse strains showed tremor with vacuole formation $[16,28]$. However, the Aspa-knockout rats produced in this study showed no tremor despite the formation of vacuoles.

Involvement of Aspa in development of essential tremor

We obtained $131 \mathrm{~F}_{2}$ progeny from intercrosses of $\left(\mathrm{WTC} / \mathrm{Kyo} \times \mathrm{F} 344-\right.$ Aspa $^{\text {em34Kyo }}$ ) $\mathrm{F}_{1}$ hybrids. We genotyped them for the 16-bp deletion in the Aspa genes and the $\mathrm{A} 354 \mathrm{~V}$ missense mutation in the $\mathrm{Hcn} 1$ gene and found that nine rats (male $=4$ and female $=5$ ) were homozygous for both the Aspa deletion and Hcnl missense mutation. The Aspa/Hcnl double-mutant rats showed spontaneous tremor after weaning. There was no sex difference in intensity or duration of the tremor. The tremors in these rats appeared during movement inde- 
pendent of space and time and were especially prominent in the forelimbs, upper trunk, head, and neck (Supplementary Video 1). These tremors appeared similar to those found in TRM/Kyo rats. The rats having other genotypes, including rats homozygous for the Aspa deletion or homozygous for the Hcnl missense mutation, showed no tremor.

To evaluate the pharmacological responses of the Aspa/Hcn 1 double-mutant rats to anti-tremor drugs, we used drugs therapeutic for either ET (i.e., propranolol and phenobarbital) or Parkinson's disease tremor (i.e., trihexyphenidyl). Propranolol and phenobarbital significantly inhibited tremor duration $(F=27.2, \mathrm{df}=4$, $P<0.01$ for propranolol; $F=16.8, \mathrm{df}=4, P<0.01$ for phenobarbital) and intensity $\left(\chi^{2}=24.8, \mathrm{df}=4 P<0.01\right.$ for propranolol; $\chi^{2}=23.0, \mathrm{df}=4 \quad P<0.01$ for phenobarbital). By contrast, the antiparkinsonian agent trihexyphenidyl failed to affect tremor (Fig. 3A).

These findings indicated that the tremors observed in the Aspa/Hcn 1 double-mutant rats were equivalent to those in TRM/Kyo rats and strongly suggested that the lack of the Aspa gene together with the Hcnl gene mutation was causative for tremor expression (Fig. 3B).

\section{Discussion}

In the present study, we demonstrated that the knocked out Aspa allele induced tremor when it was combined with the $\mathrm{Hcnl}$ loss-of-function missense mutation $(\mathrm{Hc}$ $n 1^{A 354 V}$ ). Tremors in the Aspa/Hcnl double-mutant rats were similar to those in the TRM/Kyo rats and were selectively suppressed by drugs that treat ET but not those that treat parkinsonism. These findings clearly indicated that the lack of the Aspa gene, in addition to the Hcnl gene mutation, was essential for tremor expression in a rat model of ET and suggested the potential for an alteration of the human ASPA gene in ET.

To date, genetic studies with ET families have identified several loci and genes that are associated with ET [11]. However, mutations in the human ASPA gene (17p13.2) have not been found in ET patients, and the locus is not included in the ET-associated loci $[6,7,26]$. Given that the ASPA defect could confer a tremor phenotype when it is combined with the HCN1 defect, the digenic inheritance model, or two-locus model, can be applied to ET genetics; and, thereby, mutations at the ASPA locus may be identified [24].

Our findings indicated that the combination of the
Aspa and Hcnl genes was important for the development of tremors, providing insights into tremor genesis in a rat model. Aspa encodes aspartoacylase (EC 3.5.1.15), which hydrolyzes NAA to aspartate and acetate. A defect in the ASPA function causes accumulation of NAA and depletion of acetate in the CNS. It has been proposed that NAA is associated with brain water homeostasis by regulating osmotic balance [2]. NAA and $N$-acetylaspartylglutamic acid (NAAG), which is synthesized from NAA and glutamate, function as excitatory neurotransmitters by acting on metabotropic glutamate receptors [29]. In addition, acetate is necessary for myelin lipid synthesis. Thus, it is likely that disturbance of the osmotic regulation, accumulation of neurotransmitters, and depletion of lipid precursors may occur in the brain of the Aspa-knockout rat.

$\mathrm{Hcn} 1$ encodes one of the four isoforms of the HCN channel family (HCN1-4), which are permeable to sodium and potassium ions and are activated by membrane hyperpolarization. In the brain, $\mathrm{HCN} 1$ channels conduct hyperpolarization-activated current $\left(I_{h}\right)$, which contributes to spontaneous rhythmic activity and stabilization of the neuronal membrane potential against excitatory or inhibitory inputs $[13,20]$. HCN1 channels are expressed densely in the inferior olive [20]. The $I_{h}$ in the inferior olive is transmitted to the cerebellar via climbing fibers and is involved in controlling and coordinating movements, possibly including body tremor [13]. When $I_{h}$ are blocked, inferior olive neurons hyperpolarize, and robust (abnormal) rhythmic oscillations are evoked [1]. Thus, it is likely that disturbance of the $I_{h}$ and abnormal oscillations may occur in the brain of the Hcn $1^{A 354 V_{-}}$ mutant rat. Therefore, we speculate that the neuronal excitation due to abundant NAA and NAAG and the abnormal oscillations due to the blockage of the $I_{h}$ mainly in the olivocerebellar pathway confer tremor development in Aspa/Hcn1 double-mutant rats.

The Aspa-knockout rats we produced in the present study showed no tremor until the Hcnl mutation was introduced. Such phenotypic differences in tremor expression in the ASPA defect have also been observed in Aspa-knockout mice. To date, various Aspa-knockout mouse strains have been produced and some show tremor but others do not [18]. Thus, it is likely that tremor expression under the ASPA defect may be strongly affected by other genetic factors, rather than by a species difference.

The Aspa-knockout rat has the potential to serve as an 
A Propranolol $30 \mathrm{mg} / \mathrm{kg}(\mathrm{ip})$
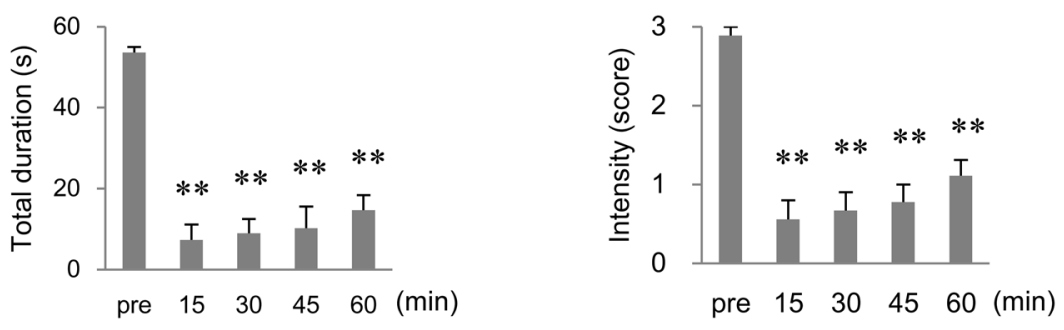

\section{Phenobarbital $20 \mathrm{mg} / \mathrm{kg}$ (ip)}
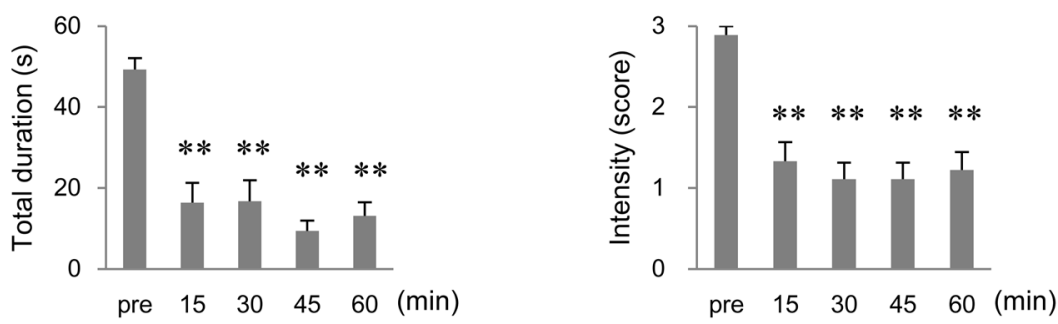

\section{Trihexyphenidyl 3 mg/kg (ip)}
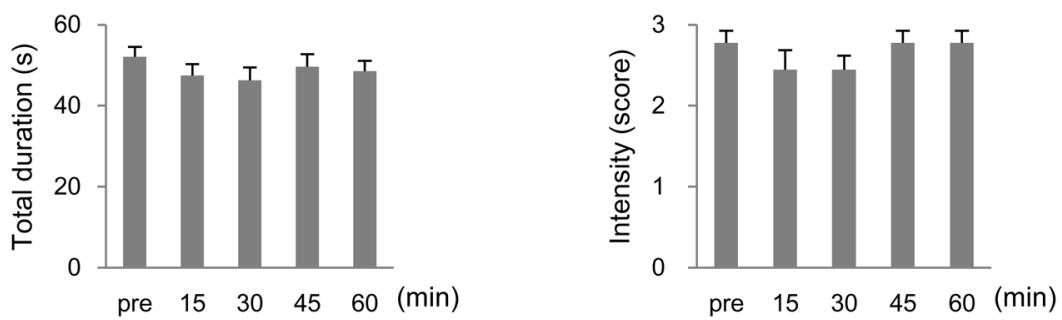

B

Hen1A354V/A354V
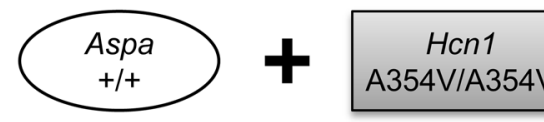

C No tremor

Aspa-/-
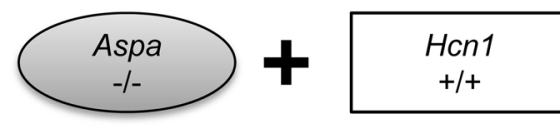

c No tremor

\section{Aspa/Hen1 double mutant}
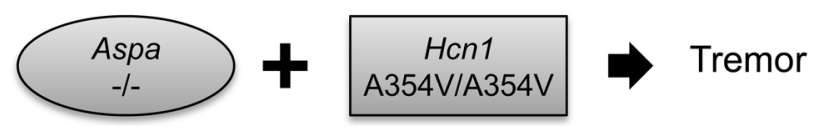

Fig. 3. Effects of anti-tremor agents on Aspa/Hcnl double-mutant rats. A, Effects of anti-tremor agents on tremor in the Aspal Hcn 1-double mutant rats. Data are presented as the mean \pm SEM of nine animals. $* * P<0.01$, vs. pre-drug control levels (pre). B, Involvement of Aspa and Hcnl in the expression of essential tremor in rats. Among F2 animals obtained from an intercross between $\left(\mathrm{WTC} / \mathrm{Kyo} \times \mathrm{F} 344-A s p a^{e m 34 K y o}\right) \mathrm{F} 1$ hybrids, rats homozygous for the Hcn 1 missense mutation or rats homozygous for the Aspa deletion show no tremor. By contrast, rats homozygous for both mutations show tremors.

animal model of Canavan disease (CD). CD is a rare human autosomal recessive genetic disorder resulting from mutations in the ASPA gene. Symptoms of CD becoming apparent in early infancy include loss of motor skills, loss of muscle control, blindness, megalocephaly, and epileptic seizures, with death usually occurring within the first decade of life [5]. The neuropathological changes include hypomyelination, leukodystrophy, and spongy degeneration in the CNS. Treatments of CD have been attempted with gene transfer, enzyme replacement, 
lithium citrate, or glyceryl triacetate $[3,25,27,30]$, but no effective treatment has been established. The Aspaknockout rat shares some phenotypes with $\mathrm{CD}$, such as spongiform degeneration, abnormal myelination, and elevated urine NAA levels. Thus, the Aspa-knockout rat can contribute to the understanding of mechanisms underlying the pathogenesis of $\mathrm{CD}$ and the development of effective prevention and treatment of $\mathrm{CD}$.

In summary, we showed that simultaneous mutation of both Aspa and Hcnl genes was essential for tremor expression in the TRM/Kyo rat. The Aspa/Hcnl-double mutant and Aspa-knockout rats can serve as good animal models of human ET and $\mathrm{CD}$, respectively.

\section{Acknowledgments}

This work was supported in part by the Japan Society for the Promotion of Science grant-in-aid 26290031. We are thankful to the National BioResource Project - Rat (NBRP-Rat) for providing WTC/Kyo rats. F344-Aspaem31 Kyo and F344-Aspa ${ }^{\text {em34Kyo }}$ rats were deposited at NBRPRat and can be provided by the resource center.

\section{References}

1. Bal, T. and McCormick, D.A. 1997. Synchronized oscillations in the inferior olive are controlled by the hyperpolarization-activated cation current $I_{h}$. J. Neurophysiol. 77: 3145-3156. [Medline]

2. Baslow, M.H. 1999. Molecular water pumps and the aetiology of Canavan disease: a case of the sorcerer's apprentice. J. Inherit. Metab. Dis. 22: 99-101. [Medline] [CrossRef]

3. Baslow, M.H. and Guilfoyle, D.N. 2013. Canavan disease, a rare early-onset human spongiform leukodystrophy: insights into its genesis and possible clinical interventions. Biochimie 95: 946-956. [Medline] [CrossRef]

4. Benito-León, J. and Louis, E.D. 2006. Essential tremor: emerging views of a common disorder. Nat. Clin. Pract. Neurol. 2: 666-678, quiz 2p, 691. [Medline] [CrossRef]

5. Gordon, N. 2001. Canavan disease: a review of recent developments. Eur. J. Paediatr. Neurol. 5: 65-69. [Medline] [CrossRef]

6. Gulcher, J.R., Jónsson, P., Kong, A., Kristjánsson, K., Frigge, M.L., Kárason, A., Einarsdóttir, I.E., Stefánsson, H., Einarsdóttir, A.S., Sigurthoardóttir, S., Baldursson, S., Björnsdóttir, S., Hrafnkelsdóttir, S.M., Jakobsson, F., Benedickz, J., and Stefánsson, K. 1997. Mapping of a familial essential tremor gene, FET1, to chromosome 3q13. Nat. Genet. 17: 84-87. [Medline] [CrossRef]

7. Higgins, J.J., Pho, L.T., and Nee, L.E. 1997. A gene (ETM) for essential tremor maps to chromosome 2p22-p25. Mov. Disord. 12: 859-864. [Medline] [CrossRef]

8. Ishishita, S., Inui, T., Matsuda, Y., Serikawa, T., and Kitada,
K. 2013. Infertility associated with meiotic failure in the tremor rat $(\mathrm{tm} / \mathrm{tm})$ is caused by the deletion of spermatogenesis associated 22. Exp. Anim. 62: 219-227. [Medline] [CrossRef]

9. Jiménez-Jiménez, F.J., Alonso-Navarro, H., García-Martín, E., Lorenzo-Betancor, O., Pastor, P., and Agúndez, J.A. 2013. Update on genetics of essential tremor. Acta Neurol. Scand. 128: 359-371. [Medline] [CrossRef]

10. Kuhara, T., Inoue, Y., Ohse, M., Krasnikov, B.F., and Cooper, A.J. 2011. Urinary 2-hydroxy-5-oxoproline, the lactam form of $\alpha$-ketoglutaramate, is markedly increased in urea cycle disorders. Anal. Bioanal. Chem. 400: 1843-1851. [Medline] [CrossRef]

11. Kuhlenbäumer, G., Hopfner, F., and Deuschl, G. 2014. Genetics of essential tremor: meta-analysis and review. Neurology 82: 1000-1007. [Medline] [CrossRef]

12. Kuramoto, T., Kuwamura, M., Tokuda, S., Izawa, T., Nakane, Y., Kitada, K., Akao, M., Guénet, J.L., and Serikawa, T. 2011. A mutation in the gene encoding mitochondrial $\mathrm{Mg}^{2+}$ channel MRS2 results in demyelination in the rat. PLoS Genet. 7: e1001262. [Medline] [CrossRef]

13. Llinás, R. and Yarom, Y. 1986. Oscillatory properties of guinea-pig inferior olivary neurones and their pharmacological modulation: an in vitro study. J. Physiol. 376: 163-182. [Medline] [CrossRef]

14. Louis, E.D. 2001. Etiology of essential tremor: should we be searching for environmental causes? Mov. Disord. 16: 822-829. [Medline] [CrossRef]

15. Mashimo, T., Kaneko, T., Sakuma, T., Kobayashi, J., Kunihiro, Y., Voigt, B., Yamamoto, T., and Serikawa, T. 2013. Efficient gene targeting by TAL effector nucleases coinjected with exonucleases in zygotes. Sci. Rep. 3: 1253. [Medline] [CrossRef]

16. Matalon, R., Rady, P.L., Platt, K.A., Skinner, H.B., Quast, M.J., Campbell, G.A., Matalon, K., Ceci, J.D., Tyring, S.K., Nehls, M., Surendran, S., Wei, J., Ezell, E.L., and Szucs, S. 2000. Knock-out mouse for Canavan disease: a model for gene transfer to the central nervous system. J. Gene Med. 2: 165-175. [Medline] [CrossRef]

17. Mersmann, N., Tkachev, D., Jelinek, R., Röth, P.T., Möbius, W., Ruhwedel, T., Rühle, S., Weber-Fahr, W., Sartorius, A., and Klugmann, M. 2011. Aspartoacylase-lacZ knockin mice: an engineered model of Canavan disease. PLOS ONE 6: e20336. [Medline] [CrossRef]

18. Mouse Genome Informatics website. The Jackson Laboratory, Bar Harbor, Maine, USA. Available from http://www. informatics.jax.org [accessed January 2016].

19. Nakanishi, S., Kuramoto, T., and Serikawa, T. 2009. Simple genotyping method using Ampdirect plus and FTA technologies: application to the identification of trangenic animals and their rutine genetic monitoring. Lab. Anim. Res 25: $75-78$.

20. Notomi, T. and Shigemoto, R. 2004. Immunohistochemical localization of $I_{h}$ channel subunits, HCN1-4, in the rat brain. J. Comp. Neurol. 471: 241-276. [Medline] [CrossRef]

21. Ohno, Y., Shimizu, S., Tatara, A., Imaoku, T., Ishii, T., Sasa, M., Serikawa, T., and Kuramoto, T. 2015. Hcnl is a tremorgenic genetic component in a rat model of essential tremor. 
PLoS ONE 10: e0123529. [Medline] [CrossRef]

22. Sakuma, T., Hosoi, S., Woltjen, K., Suzuki, K., Kashiwagi, K., Wada, H., Ochiai, H., Miyamoto, T., Kawai, N., Sasakura, Y., Matsuura, S., Okada, Y., Kawahara, A., Hayashi, S., and Yamamoto, T. 2013. Efficient TALEN construction and evaluation methods for human cell and animal applications. Genes Cells 18: 315-326. [Medline] [CrossRef]

23. Sakuma, T., Ochiai, H., Kaneko, T., Mashimo, T., Tokumasu, D., Sakane, Y., Suzuki, K., Miyamoto, T., Sakamoto, N., Matsuura, S., and Yamamoto, T. 2013. Repeating pattern of non-RVD variations in DNA-binding modules enhances TALEN activity. Sci. Rep. 3: 3379. [Medline] [CrossRef]

24. Schäffer, A.A. 2013. Digenic inheritance in medical genetics. J. Med. Genet. 50: 641-652. [Medline] [CrossRef]

25. Segel, R., Anikster, Y., Zevin, S., Steinberg, A., Gahl, W.A., Fisher, D., Staretz-Chacham, O., Zimran, A., and Altarescu, G. 2011. A safety trial of high dose glyceryl triacetate for Canavan disease. Mol. Genet. Metab. 103: 203-206. [Medline] [CrossRef]

26. Shatunov, A., Sambuughin, N., Jankovic, J., Elble, R., Lee, H.S., Singleton, A.B., Dagvadorj, A., Ji, J., Zhang, Y., Ki- monis, V.E., Hardy, J., Hallett, M., and Goldfarb, L.G. 2006. Genomewide scans in North American families reveal genetic linkage of essential tremor to a region on chromosome 6p23. Brain 129: 2318-2331. [Medline] [CrossRef]

27. Solsona, M.D., Fernández, L.L., Boquet, E.M., and Andrés, J.L. 2012. Lithium citrate as treatment of Canavan disease. Clin. Neuropharmacol. 35: 150-151. [Medline] [CrossRef]

28. Traka, M., Wollmann, R.L., Cerda, S.R., Dugas, J., Barres, B.A., and Popko, B. 2008. Nur7 is a nonsense mutation in the mouse aspartoacylase gene that causes spongy degeneration of the CNS. J. Neurosci. 28: 11537-11549. [Medline] [CrossRef]

29. Yan, H.D., Ishihara, K., Serikawa, T., and Sasa, M. 2003. Activation by $\mathrm{N}$-acetyl-${ }_{\mathrm{L}}$-aspartate of acutely dissociated hippocampal neurons in rats via metabotropic glutamate receptors. Epilepsia 44: 1153-1159. [Medline] [CrossRef]

30. Zano, S., Malik, R., Szucs, S., Matalon, R., and Viola, R.E. 2011. Modification of aspartoacylase for potential use in enzyme replacement therapy for the treatment of Canavan disease. Mol. Genet. Metab. 102: 176-180. [Medline] [CrossRef] 